\title{
Self Replication and Signalling
}

\author{
I.Chakrabarty $^{a}$, Prashant $^{b}$, B.S.Choudhury ${ }^{c}$ \\ ${ }^{a}$ Department of Mathematics, \\ Heritage Institute of Technology,Kolkata-107,West Bengal,India \\ ${ }^{b}$ Indian Institute of Information Technology and Management, Gwalior,MP, India \\ ${ }^{c}$ Department of Mathematics \\ Bengal Engineering and Science University, Howrah, India
}

\begin{abstract}
It is known that if one could clone an arbitrary quantum state one could send signal faster than the speed of light. However it remains interesting to see that if one can perfectly self replicate an arbitrary quantum state, does it violate the no signalling principle? Here we see that perfect self replication would also lead to superluminal signalling.
\end{abstract}

\section{Introduction}

It was Shannon who first introduced us to the amazing world of 0 and 1 . After that it had been a long journey and still we are searching for the nature of information in quantum world. One of the major thrust area of research is to look out how the information defined in classical world differs from quantum information and also to enlist many computational procedures which are feasible in the classical world but get restricted at microscopic level. Cloning and deletion which is feasible in classical information, cannot be executed with

\footnotetext{
${ }^{*}$ Corresponding author: E-Mail-indranilc@indiainfo.com
} 
hundred percent fidelity for an unknown quantum state $[1,2]$. Recently it had been seen that self replication machine which is feasible in classical information theory [3] fails to self replicate two non orthogonal quantum states [4]. It may be remarked that the process of replication or cloning and self replication is not just the same thing. For the proper understanding of the matter we are introducing the concept of universal constructor.

A Universal Constructor: A Universal constructor or a quantum mechanical self replicating machine is defined as a machine which can implement the copying of the original state along with the stored programme by a linear operator acting on the joint Hilbert Space. This machine may be completely specified by a quadruple $\left(|\psi\rangle,\left|P_{U}\right\rangle,|C\rangle,|\Sigma\rangle\right)$. Here $|\psi\rangle \in H^{N}$ (where $H$ is the associated Hilbert space with dimension 'N') is the input state that contains quantum information to be self replicated and $\left|P_{U}\right\rangle \in H^{K}$ (where $K$ is the dimension of the associated Hilbert space)is the programme state that carries the instructions to copy the original information (the unitary operator $U ; U(|\psi\rangle|0\rangle)=|\psi\rangle|\psi\rangle$, is encoded in the programme state $\left.\left|P_{U}\right\rangle\right)$. Here $|C\rangle$ is the control qubit, which separates the original information from the program states so that there remains no quantum entanglement between the replicated and the original information. $|\Sigma\rangle$ is the finite collection of n blank states $|0\rangle|0\rangle|0\rangle \ldots . . .|0\rangle$ in a $M$ dimensional Hilbert space, where each $|0\rangle \in H^{N}$. Hence $M=N^{n}$. In order to copy the program states say $m$ blank states are needed, so $K=N^{m}$ We had already seen in $[4,5]$ that self replication of two non-orthogonal programme state is not feasible in general. However for self replication of a finite number $\mathrm{K}$ of the non-orthogonal states with $\mathrm{K}$ the dimension of the program Hilbert space, then it may be possible to design a universal constructor with finite resources.

In this work we try to see that whether the principle of no self replication is consistent with the principle of no signalling. The principle of no signalling states that : If two parties (say Alice and Bob) are separated by space like distance, then one cannot send superluminal signal faster than the speed of light. All the no go theorems like cloning, deletion, flipping are consistent with this principle of no signalling $[6,7,8]$. Here we will see that 'no-self replication' theorem is also consistent with the principle of no signalling. In this problem we will consider a hypothetical situation where two distant parties Alice and Bob share an entangled state. Now if Alice self replicates her qubit with the help 
of a universal constructor, then Alice can distinguish two statistical mixture representing her subsystem as a consequence of Bob's measurement on two different qubit basis. As a result of which Alice would tell on which basis Bob has measured his qubit. This will imply superluminal signalling. Interestingly in the next section we are going to show that this is not going to be true.

\section{Principle of no signalling and self replication :}

Let us consider two entangled states shared by two distant partners Alice and Bob. The entangled states are given as

$$
\begin{array}{r}
\left|\chi_{1}\right\rangle=\frac{1}{\sqrt{2}}\left[\left|\psi_{1}\right\rangle_{A}\left|\psi_{2}\right\rangle_{B}-\left|\psi_{2}\right\rangle_{A}\left|\psi_{1}\right\rangle_{B}\right] \\
\left|\chi_{2}\right\rangle=\frac{1}{\sqrt{2}}\left[\left|P_{u 1}\right\rangle_{A}\left|P_{u 2}\right\rangle_{B}-\left|P_{u 2}\right\rangle_{A}\left|P_{u 1}\right\rangle_{B}\right]
\end{array}
$$

where $A$ denotes that the qubit is in possession with Alice, and $B$ denotes Bob's qubit. The states $\left|\psi_{1}\right\rangle,\left|\psi_{2}\right\rangle$ are two non orthogonal quantum states whereas the states $\left|P_{u 1}\right\rangle,\left|P_{u 2}\right\rangle$ are non orthogonal quantum states. The combined state of Alice and bob is given by,

$$
\begin{aligned}
\left|\chi_{12}\right\rangle= & \frac{1}{2}\left[\left(\left|\psi_{1}\right\rangle\left|P_{u 1}\right\rangle\right)_{A}\left(\left|\psi_{2}\right\rangle\left|P_{u 2}\right\rangle\right)_{B}-\left(\left|\psi_{2}\right\rangle\left|P_{u 1}\right\rangle\right)_{A}\left(\left|\psi_{1}\right\rangle\left|P_{u 2}\right\rangle\right)_{B}-\right. \\
& \left.\left(\left|\psi_{1}\right\rangle\left|P_{u 2}\right\rangle\right)_{A}\left(\left|\psi_{2}\right\rangle\left|P_{u 1}\right\rangle\right)_{B}-\left(\left|\psi_{2}\right\rangle\left|P_{u 2}\right\rangle\right)_{A}\left(\left|\psi_{1}\right\rangle\left|P_{u 1}\right\rangle\right)_{B}\right]
\end{aligned}
$$

The principle of no signalling tells us that if some local operation is done on Bob's qubit ,it will not change the density matrix describing Alice's subsystem. Now , in this context , where Alice and Bob are sharing entangled states (2), if we trace out Bob's qubit, the density matrix describing Alice's subsystem is given by,

$$
\begin{aligned}
\rho_{A}= & \frac{1}{4}\left[\left|\psi_{1} P_{u 1}\right\rangle\left\langle\psi_{1} P_{u 1}|+| \psi_{2} P_{u 1}\right\rangle\left\langle\psi_{2} P_{u 1}|+| \psi_{1} P_{u 2}\right\rangle\left\langle\psi_{1} P_{u 2}|+| \psi_{2} P_{u 2}\right\rangle\left\langle\psi_{2} P_{u 2}\right|\right. \\
& -\left|\psi_{2} P_{u 1}\right\rangle\left\langle\psi_{1} P_{u 1}\left|\left(\left\langle\psi_{2} \mid \psi_{1}\right\rangle\right)-\right| \psi_{1} P_{u 2}\right\rangle\left\langle\psi_{1} P_{u 1}\right|\left(\left\langle P_{u 2} \mid P_{u 1}\right\rangle\right) \\
& +\left|\psi_{2} P_{u 2}\right\rangle\left\langle\psi_{1} P_{u 1}\left|\left(\left\langle\psi_{2} \mid \psi_{1}\right\rangle\left\langle P_{u 2} \mid P_{u 1}\right\rangle\right)-\right| \psi_{1} P_{u 1}\right\rangle\left\langle\psi_{2} P_{u 1}\right|\left(\left\langle\psi_{1} \mid \psi_{2}\right\rangle\right) \\
& +\left|\psi_{1} P_{u 2}\right\rangle\left\langle\psi_{2} P_{u 1}\left|\left(\left\langle\psi_{1} \mid \psi_{2}\right\rangle\left\langle P_{u 2} \mid P_{u 1}\right\rangle\right)-\right| \psi_{2} P_{u 2}\right\rangle\left\langle\psi_{2} P_{u 1}\right|\left(\left\langle P_{u 2} \mid P_{u 1}\right\rangle\right) \\
& -\left|\psi_{1} P_{u 1}\right\rangle\left\langle\psi_{1} P_{u 2}\left|\left(\left\langle P_{u 1} \mid P_{u 2}\right\rangle\right)+\right| \psi_{2} P_{u 1}\right\rangle\left\langle\psi_{1} P_{u 2}\right|\left(\left\langle\psi_{2} \mid \psi_{1}\right\rangle\left\langle P_{u 1} \mid P_{u 2}\right\rangle\right) \\
& -\left|\psi_{2} P_{u 2}\right\rangle\left\langle\psi_{1} P_{u 2}\left|\left(\left\langle\psi_{1} \mid \psi_{2}\right\rangle\right)+\right| \psi_{1} P_{u 1}\right\rangle\left\langle\psi_{2} P_{u 2}\right|\left(\left\langle\psi_{1} \mid \psi_{2}\right\rangle\left\langle P_{u 1} \mid P_{u 2}\right\rangle\right)
\end{aligned}
$$




$$
\left.-\left|\psi_{2} P_{u 1}\right\rangle\left\langle\psi_{2} P_{u 2}\left|\left(\left\langle P_{u 1} \mid P_{u 2}\right\rangle\right)-\right| \psi_{1} P_{u 2}\right\rangle\left\langle\psi_{2} P_{u 2}\right|\left(\left\langle\psi_{1} \mid \psi_{2}\right\rangle\right)\right]
$$

At this point one can ask an interesting question that if Bob can self replicate his qubit, then will there be any change in the density matrix (3) describing Alice's subsystem. The principle of no signalling tells us that it is impossible to achieve.

Let us Bob possesses a hypothetical quantum mechanical universal constructor, which will self replicate the qubit possessed by Bob. The action of the self replicating machine is given by,

$$
\begin{aligned}
& \left|\psi_{1}\right\rangle|0\rangle\left|P_{u_{1}}\right\rangle|0\rangle^{\otimes m}|C\rangle|0\rangle^{\otimes n-(m+1)} \longrightarrow\left|\psi_{1}\right\rangle\left|P_{u_{1}}\right\rangle\left(\left|\psi_{1}\right\rangle|0\rangle\left|P_{u_{1}}\right\rangle|0\rangle^{\otimes m}\left|C_{1}\right\rangle\right)|0\rangle^{\otimes n-2(m+1)} \\
& \left|\psi_{2}\right\rangle|0\rangle\left|P_{u_{1}}\right\rangle|0\rangle^{\otimes m}|C\rangle|0\rangle^{\otimes n-(m+1)} \longrightarrow\left|\Phi_{1}\right\rangle \\
& \left|\psi_{1}\right\rangle|0\rangle\left|P_{u_{2}}\right\rangle|0\rangle^{\otimes m}|C\rangle|0\rangle^{\otimes n-(m+1)} \longrightarrow\left|\Phi_{2}\right\rangle \\
& \left|\psi_{2}\right\rangle|0\rangle\left|P_{u_{2}}\right\rangle|0\rangle^{\otimes m}|C\rangle|0\rangle^{\otimes n-(m+1)} \longrightarrow\left|\psi_{2}\right\rangle\left|P_{u_{2}}\right\rangle\left(\left|\psi_{2}\right\rangle|0\rangle\left|P_{u_{2}}\right\rangle|0\rangle^{\otimes m}\left|C_{2}\right\rangle\right)|0\rangle^{\otimes n-2(m+1}(4)
\end{aligned}
$$

Here $\left|P_{u_{1}}\right\rangle$ and $\left|P_{u_{2}}\right\rangle$ are the program states where the instruction to copy the original information has been encoded. Here the state $|C\rangle$ acts as an control unit which separates the original with the replica in such a manner so that there exists no quantum entanglement between them. Here $\left|C_{1}\right\rangle$ and $\left|C_{2}\right\rangle$ are the respective states of the control unit at the output port. The states $\left|\Phi_{1}\right\rangle$ and $\left|\Phi_{2}\right\rangle$ are the composite states at the output port. It is important to note that the above transformations (2-3)are not merely a cloning transformation, on the contrary it is a recursively defined transformation where the fixed unitary operator acts on initial configuration and the same operator acts on the final configuration after the copies have been produced.

Now Bob attaches ancilla states $|\Sigma\rangle=|0\rangle^{\otimes n}$ as well as the control qubit $|C\rangle$ to his subsystem. Now Bob applies the transformations defined in (3) on his qubit, in such a way that out of these $n$ blank states he uses only one blank state for the cloning of the original states, m blank states for the cloning of the programmed states and he is left with $n-(m+1)$ blank states for future replication, until all the $\mathrm{n}$ blank states are exhausted. He also uses the control qubit to ensure that there remains no entanglement between the original and replica. As result of his action the entangled state shared between these two parties Alice and Bob takes the form,

$$
\left|\chi_{12}^{S}\right\rangle=\frac{1}{2}\left[\left(\left|\psi_{1}\right\rangle\left|P_{u 1}\right\rangle\right)|Y\rangle-\left(\left|\psi_{2}\right\rangle\left|P_{u 1}\right\rangle\right)\left|\Phi_{1}\right\rangle-\left(\left|\psi_{1}\right\rangle\left|P_{u 2}\right\rangle\right)\left|\Phi_{2}\right\rangle+\left(\left|\psi_{2}\right\rangle\left|P_{u 2}\right\rangle\right)|X\rangle\right]
$$


where,

$|Y\rangle=\left|\psi_{2}\right\rangle\left|P_{u_{2}}\right\rangle\left(\left|\psi_{2}\right\rangle|0\rangle\left|P_{u_{2}}\right\rangle|0\rangle^{\otimes m}\left|C_{2}\right\rangle\right)|0\rangle^{\otimes n-2(m+1)}$

$|X\rangle=\left|\psi_{1}\right\rangle\left|P_{u_{1}}\right\rangle\left(\left|\psi_{1}\right\rangle|0\rangle\left|P_{u_{1}}\right\rangle|0\rangle^{\otimes m}\left|C_{1}\right\rangle\right)|0\rangle^{\otimes n-2(m+1)}$

Now if one traces out Bob's qubit, then the density matrix representing Alice's subsystem is given by,

$$
\begin{aligned}
\rho_{A}^{S} & =\frac{1}{4}\left[\left|\psi_{1} P_{u 1}\right\rangle\left\langle\psi_{1} P_{u 1}|+| \psi_{1} P_{u 2}\right\rangle\left\langle\psi_{1} P_{u 2}|+| \psi_{2} P_{u 1}\right\rangle\left\langle\psi_{2} P_{u 1}|+| \psi_{2} P_{u 2}\right\rangle\left\langle\psi_{2} P_{u 2}\right|\right. \\
& +\left|\psi_{2} P_{u 2}\right\rangle\left\langle\psi_{1} P_{u 1}|(\langle Y \mid X\rangle)-| \psi_{2} P_{u 1}\right\rangle\left\langle\psi_{1} P_{u 1}\left|\left(\left\langle Y \mid \Phi_{1}\right\rangle\right)-\right| \psi_{1} P_{u 2}\right\rangle\left\langle\psi_{1} P_{u 1}\right|\left(\left\langle Y \mid \Phi_{2}\right\rangle\right) \\
& -\left|\psi_{1} P_{u 1}\right\rangle\left\langle\psi_{2} P_{u 1}\left|\left(\left\langle\Phi_{1} \mid Y\right\rangle\right)+\right| \psi_{1} P_{u 2}\right\rangle\left\langle\psi_{2} P_{u 1}\left|\left(\left\langle\Phi_{1} \mid \Phi_{2}\right\rangle\right)-\right| \psi_{2} P_{u 2}\right\rangle\left\langle\psi_{2} P_{u 1}\right|\left(\left\langle\Phi_{1} \mid X\right\rangle\right) \\
& -\left|\psi_{1} P_{u 1}\right\rangle\left\langle\psi_{1} P_{u 2}\left|\left(\left\langle\Phi_{2} \mid Y\right\rangle\right)+\right| \psi_{2} P_{u 1}\right\rangle\left\langle\psi_{1} P_{u 2}\left|\left(\left\langle\Phi_{2} \mid \Phi_{1}\right\rangle\right)-\right| \psi_{2} P_{u 2}\right\rangle\left\langle\psi_{1} P_{u 2}\right|\left(\left\langle\Phi_{2} \mid X\right\rangle\right) \\
& \left.\left.-\left|\psi_{2} P_{u 1}\right\rangle\left\langle\psi_{2} P_{u 2}\left|\left(\left\langle X \mid \Phi_{1}\right\rangle\right)-\right| \psi_{1} P_{u 2}\right\rangle\left\langle\psi_{2} P_{u 2}\left|\left(\left\langle X \mid \Phi_{2}\right\rangle\right)+\right| \psi_{1} P_{u 1}\right\rangle\left\langle\psi_{2} P_{u 2}\right|(\langle X \mid Y\rangle)\right\} 6\right)
\end{aligned}
$$

Since the density matrices (3) and (6) representing Alice's subsystem before and after the application of Universal self replicating machine on his qubit by Bob are not identical, we conclude that signalling has taken place due to local action performed by Bob. However no signalling principle tells us that this is not possible in reality. Hence we conclude that perfect self replication for arbitrary quantum states is not possible from the principle of no signalling.

\section{Conclusion:}

In summary we can say that this work once again shows the peaceful coexistence of no go theorem like no self replication theorem with the physical principles like principle of no signalling. In other words, one cannot self replicate arbitrary quantum states with the help of program state as this will violate the principle of no signalling.

\section{Acknowledgement}

Indranil Chakrabarty acknowledges S. Adhikari, Prof.C.G.Chakraborti for their encouragement and inspiration in completing this work. Prashant acknowledges Almighty for 
getting the inspiration for research and also Prof Gilles Bassard for providing encouragement and support in complementing the work . Both Indranil and Prashant acknowledges Prof A.K.Pati, for providing encouragement and support in completing this work.

\section{Reference:}

[1] W.K.Wootters and W.H.Zurek,Nature 299, 802 (1982) .

[2] A.K.Pati and S.L.Braunstein Nature 404, 164 (2000) .

[3] J.Von Neumann, The theory of Self-Replicating Automata. University of Illinois Press,Urbana,IL (1966)(work by J.von Neumann in 1952-53).

[4] A.K.Pati and S.L.Braunstein, Quantum mechanical universal constructor,quantph /0303124 (2003).

[5] M. A. Nielsen, I. Chuang, Phys. Rev. Lett. 79, 321 (1997).

[6] A.K.Pati, S.L.Braunstein, Phys. Lett A 315, 208 (2003).

[7] I.Chattopadhyay.et.al.Phys. Lett. A, 351, 384 (2006).

[8] A.K.Pati, Phys. Lett. A, 292, 12 (2001). 